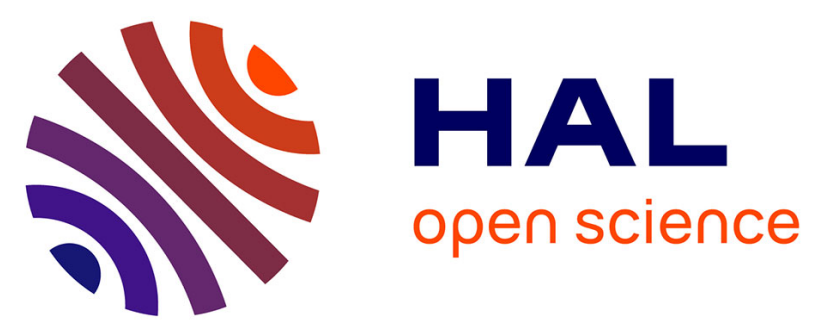

\title{
Performance analysis of cellular networks with opportunistic scheduling using queueing theory and stochastic geometry
}

\author{
Bartlomiej Blaszczyszyn, Mohamed Kadhem Karray
}

\section{To cite this version:}

Bartlomiej Blaszczyszyn, Mohamed Kadhem Karray. Performance analysis of cellular networks with opportunistic scheduling using queueing theory and stochastic geometry. IEEE Transactions on Wireless Communications, 2019, 18 (12), pp.5952-5966. 10.1109/TWC.2019.2940938 • hal-01824986v2

\section{HAL Id: hal-01824986 \\ https://hal.inria.fr/hal-01824986v2}

Submitted on 9 Sep 2019

HAL is a multi-disciplinary open access archive for the deposit and dissemination of scientific research documents, whether they are published or not. The documents may come from teaching and research institutions in France or abroad, or from public or private research centers.
L'archive ouverte pluridisciplinaire HAL, est destinée au dépôt et à la diffusion de documents scientifiques de niveau recherche, publiés ou non, émanant des établissements d'enseignement et de recherche français ou étrangers, des laboratoires publics ou privés. 


\title{
Performance analysis of cellular networks with opportunistic scheduling using queueing theory and stochastic geometry
}

\author{
Bartłomiej Błaszczyszyn*† and Mohamed Kadhem Karray ${ }^{\ddagger}$ Member, IEEE \\ *Inria-ENS, 23 Avenue d'Italie, 75214 Paris, FRANCE; Email: Bartek.Blaszczyszyn@ens.fr \\ $\dagger$ DI-ENS, CNRS, Inira, PSL Research University, Paris, France \\ $\ddagger$ Orange Labs, 44 avenue de la Rpublique, 92320 Châtillon, FRANCE; Email: mohamed.karray@orange.com
}

\begin{abstract}
Combining stochastic geometric approach with some classical results from queueing theory regarding generalized processor sharing queues, in this paper we extend the synthetic framework for the performance study of large cellular networks, previously proposed in $[1,2]$, by allowing it to take into account opportunistic scheduling. Rapid and verifiable with respect to real data, our approach is particularly useful for network dimensioning and long term economic planning. It is based on a detailed network model combining an informationtheoretic representation of the link layer, a queueing-theoretic representation of the users' scheduler, and a stochastic-geometric representation of the signal propagation and the network cells. It allows one to evaluate principal characteristics of the individual cells, such as loads the mean number of users and the user throughput. A simplified, Gaussian approximate model is also proposed to facilitate study of the spatial distribution of these metrics across the network. Using of both models requires only simulations of the point process of base stations and the shadowing field to estimate the expectations of some stochasticgeometric functionals not admitting explicit expressions. A key observation of our approach, bridging spatial and temporal analysis, relates the SINR distribution of the typical user to the load of the typical cell of the network. The former is a static characteristic of the network related to its spectral efficiency while the latter characterizes the performance of the (generalized) processor sharing queue serving the dynamic population of users of this cell.
\end{abstract}

Index Terms-user-throughput, traffic demand, cell-load equations, cellular network, opportunistic scheduling, typical cell, queueing theory, point process, ergodicity, Palm theory, Little's law, measurements

\section{INTRODUCTION}

Motivated by the increasing traffic in commercial cellular networks featuring opportunistic scheduling policies, in this paper we propose a synthetic model, based on queueing theory and stochastic geometry, allowing one to evaluate performance of such networks by some static simulations, with a particular focus on the variability of the quality of service characteristics across the network. We build on our previous work [1, 2], where a network evaluation model has been proposed, assuming a homogeneous space-time Poisson process of call arrivals served by a spatially stationary cellular network, with processor sharing queue models operating at all

This paper reports the results of a research undertaken under the contract CRE $N^{\circ}$ G10192 between Inria and Orange. network stations. These queues are related to each other via some system of cell-load equations capturing the dependence caused by the extra-cell interference, and bridging the spatial analysis of the SINR distribution of the typical user and the temporal analysis of the load of the typical cell of the network. Among principal metrics studied via the cell-load equations are cell loads (defined as the fraction of time the station is busy), mean number of users and their throughput at individual network cells. Following [2], we also propose an efficient Gaussian approximate model for the rapid study of the spatial distribution of these metrics across the network. The study of our synthetic model involves only simulations of the point process of base stations and the shadowing field to estimate the expectations of some stochastic geometric functionals not admitting explicit expressions. All lower level performance characteristics, involving the link layer and the opportunistic scheduler admit explicit expressions and need not be estimated by simulations. This approach allows one for a rapid and verifiable with respect to real data evaluation of the impact of the opportunistic scheduling on the global network performance. It is particularly useful for network dimensioning and long term economic planning.

\section{A. Contributions}

This paper contributes to the development of the synthetic framework for the performance study of large cellular networks previously proposed in $[1,2]$. The contribution consists in using generalized processor sharing queues in the medium access layer to model opportunistic scheduling implemented in operational networks. Previously considered model assumes round-robin scheduling. This contribution, summarized in Proposition 2, implies revisiting of Proposition 3, providing the mean characteristics of the main stochastic geometric model, as well as the extension of some analytic and numerical results (these latter formulated as Observations) justifying the Gaussian approximate model (Observation 2, Proposition 6, Proposition 7, Observation 3). A special effort is made to validate the proposed extension with respect to the real data collected in a reference $4 \mathrm{G}$ network featuring opportunistic scheduling, at the level of individual cells (Observation 1) and at the network level (Observation 4). 


\section{B. Paper organization}

The remaining part of the paper is organized as follows. We complete our introduction by discussing in Section I-C some related work. The detailed model is described in Section II. Theoretical analysis of this model and a Gaussian approximate model is presented in Section III. Our numerical study, including validation of the Gaussian approximate model with respect to the detailed model and with respect to real data is presented in Section IV. We recapitulate our work in Section V.

\section{Related work}

A large amount of literature has emerged on the performance analysis of cellular networks; a complete review falls out of the reach of this paper. In what follows we mention only some works that we found most relevant.

a) Pure simulation approach: Many network operators and other actors develop complex and time consuming simulation tools such as those developed by the industrial contributors to 3GPP (3rd Generation Partnership Project) [3]. There are many other simulation tools such as TelematicsLab LTESim [4], University of Vienna LTE simulator [5, 6] and LENA tool [7, 8] of CTTC, which are not necessarily compliant with 3GPP. Our approach requires only static simulations to estimate some stochastic geometric expectations with all lower level performance characteristics admitting explicit expressions. This makes it significantly more rapid.

b) Analytic approach: Most of the analytic studies of the performance of cellular networks focus on some particular layer. For example, information theoretic characterization of the individual link performance is proposed in [9-11]. Queueing theoretic modeling and analysis of the user traffic can be found e.g. in [12-17]. Following the pioneer work in [18] and [19], a large amount of literature uses stochastic geometry to build explicit expressions of the distribution of the downlink SINR at a typical location in the network; see [20, Chapters 57] for a complete treatment of this subject. Our comprehensive approach to the evaluation of large cellular networks strongly builds on these more focused studies.

c) Space-time models: While all modern stochastic geometric models of cellular networks integrate expressions inspired by information theory, using queueing theory in random geometric context is more rare and thus deserves a few comments.

Theoretical foundations of spatial Markovian queueing systems have been worked out in [21]. Among early works using this setting, in [22] blocking probabilities of the constant bitrate traffic were studied via a spatial Erlang loss formula. In [23] some decentralized congestion control schemes were studied in the context of data traffic using spatial processor sharing queues. Both papers assume spatial Markovian arrivals to some subset of the plane representing one cell of the network. Full interference from other base stations is taken into account without capturing the interaction between network cells. To the best of our knowledge, this latter idea appears for the fist time in [24], and independently, in the context of a hexagonal network model in [17]. However, no spatial stochastic geometric analysis of these equations and of the network is proposed there.
More recently and in a different context, [25] leverages stochastic geometric and queueing theoretic techniques to investigate the performance of some cooperative communications in decentralized wireless networks. In [26] the uplink of K-tier heterogeneous networks is studied using $\mathrm{M} / \mathrm{G} / 1$ queueing model in stochastic geometric setting. Delays in the heterogeneous cellular networks with spatio-temporal random arrival of traffic are studied in [27]. The authors of [28] investigate interference, queueing delay and network throughput in interference-limited networks while taking into account diverse QoS requirements. These theoretical works have not yet been compared to real data.

Our study features in particular realistic evaluation of the opportunistic scheduler and the spatial variability of the quality of service across the networks. In what follows we comment on some previous work in this matter.

d) Opportunistic schedulling: It leverages an inherent diversity of wireless networks provided by independently timevarying channels across the different users. The benefit of this diversity is exploited by scheduling transmissions to users when their instantaneous channel quality is near its best values. The diversity gain increases with the dynamic range of the fluctuations; it can be improved by some dynamic beamforming [29] in environments with little scattering and/or slow fading. Specific opportunistic schedulers for wireless systems have been proposed and studied for some thirty years. There is a large number of papers available that propose different opportunistic scheduling techniques. These range from simple heuristic algorithms to complex mathematical models; see a survey article [30] published in 2013. The aims of these techniques vary; some proposals are only designed to increase the total network capacity, while others seek to enhance QoS objectives such as throughput and fairness.

In terms of real world implementations, opportunistic schedulers started only appearing from the 3.5G (HSDPA) network technology. The current $4 \mathrm{G}$ technology leverages some opportunistic scheduling techniques to propose higher user rates. Although it is not always possible to know the precise nature of the implemented schemes, as we shall see in this paper, some aspects of this performance can be captured at the level of individual network cells by using relatively simple mathematical models of generalized processor sharing queues [12] with an appropriate scheduling gain function.

The authors of [31] evaluate some opportunistic schedulers, both numerically and analytically, under several fading distributions and bandwidth constraints. An ubiquitous assumption is that the scheduler is not aware of the interference. This reduces the scheduling gain as recently shown in [32] assuming Voronoi network model and in [33] for the bipolar network model. These studies concentrate on the user peak bit-rate and do not evaluate user throughput that depends on the network load.

e) Spatial distribution of the quality of service: User quality of service (e.g. throughput) and network performance metrics (e.g. cell loads) represent some mean characteristics evaluated locally in the network (e.g. for each cell) over some relatively short time intervals (usually one hour). For a given time interval, these characteristics can significantly 
vary across the cells, even if the user traffic can be considered as spatially homogeneous. This phenomenon, observed in data systematically collected by mobile operators [34-36] is related to the fact that real networks are not regular (hexagonal) but exhibit non-negligible variability of the size and geometry of cells, resulting from base station deployment constraints and haphazard propagation (shadowing) effects.

In stochastic-geometric models the aforementioned irregularity of the network is represented by appropriate point process (usually Poisson) representing locations of base stations and random shadowing field (often assumed log-normal). In this approach, the spatial distribution of performance metrics corresponds to the conditional expectations of the corresponding functions given a realization of the point process of base stations and a realization of the shadowing field. These expectations represent averaging over fast fading and dynamic configurations of users. The term "meta-distribution" was coined in [37] for the conditional distribution of the SINR given location of stations; see [20, Section 6.3] for more details.

Attention to these conditional expectations has been drown in [38] by showing that the spatial averaging of some locally finite network characteristics - mean local delays in the Poisson bipolar network model in the cited paper — can lead to infinite spatial averages in several practical cases, including the Rayleigh fading and positive thermal noise case. This mathematical fact says that a relatively large fraction of users experience in this network model large (but finite) local delays.

The spatial variability of the performance metrics was also considered in the studies of load balancing between cells. For example, [39] proposes an algorithm that compensates the spatial disparity of traffic demand, while [40] and [41] focus on the improvement of the performance of heterogeneous networks by elaborating different algorithms that insure energy, spectrum and load balancing.

f) Our previous work: The current approach based on the stochastic geometric study of the cell load equations was initially proposed in [1]. It has been extended allowing one to take into account heterogeneous networks (micro-macro cells) in [42] and spatial non-homogeneity in [43]. The study of the spatial distribution of the quality of service was initiated using this approach in [44] and further developed in [2], where the Gaussian approximated model was proposed. In all these works one assumes round-robin scheduler.

\section{DETAILED MODEL DESCRIPTION}

We shall now describe our space-time network model.

\section{A. Network architecture}

We describe first all static elements of the network model; they do not evolve in time.

1) Locations and transmission powers of base stations: We consider locations $\left\{X_{1}, X_{2}, \ldots\right\}$ of base stations (BS) on the plane $\mathbb{R}^{2}$ as a realization of a point process. We denote this point process by $\Phi .{ }^{1}$ We denote by $\mathbf{P}$ the probability on some

\footnotetext{
${ }^{1}$ According to the formalism of the theory of point processes (cf e.g. [45]), a point process is a random measure $\Phi=\sum_{n} \delta_{X_{n}}$, where $\delta_{x}$ denotes the Dirac measure at $x$
}

probability space on which $\Phi$ and all other random variables considered in what follows are defined and assume that under $\mathbf{P}, \Phi$ is stationary and ergodic ${ }^{2}$ with positive, finite intensity $\lambda_{\mathrm{BS}}$ (mean number of BS per unit of surface). We model by random variables $P_{n}\left(P_{n} \geq 0\right)$ the powers emitted by BS $X_{n}$. We assume that the point process $\Phi$ marked by $P_{n}$ 's is stationary and ergodic.

2) Propagation effects: The propagation loss is modeled by a deterministic path-loss function $l(x)=(K|x|)^{\beta}$, where $K>0$ and $\beta>2$ are given constants, and some random propagation effects. We split these effects into two categories conventionally called (fast) fading and shadowing. The former will be taken into account in the model at the link-layer, specifically in the peak bit-rate function, cf. Example 1. The shadowing impacts the choice of the serving BS and thus needs to be considered together with the network geometry. To this regard, we assume that the shadowing between a given station $X_{n} \in \Phi$ and all locations $y$ in the plane is modeled by some positive valued stochastic process $\mathbf{S}_{n}\left(y-X_{n}\right)$. The processes $\mathbf{S}_{n}(\cdot)$ are considered as marks of the points of $\Phi$, and together with transmission powers $P_{n}$ form marked point process $\tilde{\Phi}$, which is assumed stationary and ergodic. We do not make any particular assumption regarding the joint distribution of the shadowing field and the transmitted power $\left(\mathbf{S}_{0}(\cdot), P_{0}\right)$ of the typical base station (under Palm distribution; cf Footnote 6) except that $\mathbf{S}_{0}(y)$ are identically distributed across $y$.

The inverse of the power received at $y$ from $\mathrm{BS} X_{n}$, averaged over fast fading, denoted by $L_{X_{n}}(y)$ is given by

$$
L_{X_{n}}(y):=\frac{l\left(\left|y-X_{n}\right|\right)}{P_{n} \mathbf{S}_{n}\left(y-X_{n}\right)} .
$$

Slightly abusing the terminology, we will call $L_{X_{n}}(\cdot)$ the propagation-loss process from station $X_{n}$. Sometimes we will simplify the notation writing $L_{X}(\cdot)$ for the propagation-loss of BS $X \in \Phi$ and $P_{X}$ for its transmission power.

3) Service zones, SINR and peak bit-rates: We assume that each (potential) user located at $y$ on the plane is served by the BS offering the strongest received power among all the BS in the network. Thus, the zone served by BS $X \in \Phi$, denoted by $V(X)$, which is traditionally called the cell of $X$ (even if random shadowing makes it need not to be a polygon or even a connected set) is given by

$$
V(X)=\left\{y \in \mathbb{R}^{2}: L_{X}(y) \leq L_{Y}(y) \text { for all } Y \in \Phi\right\} .
$$

We define the (downlink) SINR at location $y \in V(X)$ (with respect to the serving $\mathrm{BS} X \in \Phi$ )

$$
\operatorname{SINR}(y, \tilde{\Phi}):=\frac{1 / L_{X}(y)}{N+\sum_{Y \in \Phi \backslash\{X\}} \varphi_{Y} / L_{Y}(y)},
$$

where $N$ is the noise power and $\varphi_{Y} \in[0,1]$ are some activity factors related to $\mathrm{BS} Y \in \Phi$, which will be specified in Section II-C2. Note, they weight in (3) the transmission powers in the interference term of the SINR to account for the fact that these BS might not transmit with their respective maximal powers $P_{Y}$. In general, we assume that $\varphi_{Y}$ are additional (not necessarily independent) marks of the point process $\Phi$. In fact,

\footnotetext{
${ }^{2}$ Stationarity means that the distribution of the process is translation invariant, while ergodicity allows to interpret some mathematical expectations as spatial averages of some network characteristics.
} 
we shall specify $\varphi_{Y}$ in Section II-C2 as some functionals of the locations of all points and other marks of the point process $\Phi$.

We assume that the peak bit-rate at location $y$, defined as the maximal download bit-rate available at location $y$ (achievable when all resources of the station serving this location are devoted to a single user located at $y$ ) is some function $R(\mathrm{SINR})$ of the $\operatorname{SINR}=\operatorname{SINR}(y, \tilde{\Phi})$ at this location. Our general approach presented in this paper does not depend on any particular form of this function. In our specific model of $4 \mathrm{G}$ network numerically studded in Section IV we express $R(\cdot)$ in relation to the information theoretic capacity of the additive white Gaussian noise (AWGN) multiple-input-multiple-output (MIMO) channel presented in the following example.

Example 1 (AWGN MIMO channel): Consider the AWGN MIMO channel with $t$ transmitting and $r$ receiving antennas. Denote by $H$ the circularly-symmetric complex Gaussian matrix (corresponding to Rayleigh fading) with $r$ lines and $t$ columns.

Proposition 1: [cf. [46, Theorem 1]] The ergodic coding capacity of the above AWGN MIMO channel is given by

$$
R_{\mathrm{MIMO}}(\xi)=W \mathbf{E}\left[\log _{2} \operatorname{det}\left(I_{r}+\frac{\xi}{t} H H^{*}\right)\right],
$$

where $H^{*}$ is the complex conjugate of $H, I_{r}$ is $r$-dimensional identity matrix, $\xi$ is the SNR, $W$ is the frequency bandwidth and the expectation $\mathbf{E}[\cdot]$ is taken with respect to $H$.

Expression in (4) is also the lower bound of the MIMO channel capacity in our network model with the extra-channel interference, when this latter is considered as a (part of the) noise and $\xi$ corresponds tho SINR; cf. [11, Corollary 1].

4) Scheduling gain: We consider variable bit-rate (VBR) traffic; i.e., users arrive to the network (in a way to be described in Section II-B1) and request transmissions of some data volumes at bit-rates decided by the network. When several users are present in a cell, they need to share the serving BS resources, which means that in general they do not receive their full peak bit-rates. In this paper we assume that when $n$ users are present in the cell then each of them receives a fraction $g(n) / n$ of its own peak bit-rate, i.e., the service rate $R(\mathrm{SINR}) g(n) / n$. Such a service discipline is called generalized processor sharing with scheduling gain $g(n)$. In what follows we will always assume that $g(n)>0$ for $n \geq 1$. This assumption simplifies the presentation of the stationary distribution of the processor sharing queue, cf. Section II-C1. It is also enough for our application.

Example 2 (Round robin system): In this case $g(n) \equiv 1$. This is the usual processor sharing assumption.

If $g(n)>1$ then the service rates of all users are higher than in the round robin case. This is a natural assumption in the context of this paper, where higher service rates arise as a consequence of an opportunistic scheduling of the packet transmissions to different users. This also explains why we call $g(\cdot)$ the scheduling gain. ${ }^{3}$

\footnotetext{
${ }^{3}$ If one wants to preserve the interpretation of the peak bit-rate $R$ (SINR) as the maximal rate, available when one user is served, then $g(n) \leq n$ with $g(1)=1$.
}

Example 3 (Opportunistic channel scheduling): The following function was proposed in [12] to model the performance of some opportunistic channel scheduling for cellular networks

$$
g(n)=1+1 / 2+\ldots+1 / n, \quad n \geq 1 .
$$

Note that in this case the scheduling gain $g(n)$ increases to infinity with $n$. Mathematical justification of this function requires the linear dependence of the bit-rate on independent, time-varying Rayleigh fading and user transmissions scheduled at times when they observe their best channel fading conditions. The linear dependence of a realistic peak bitrate function $R(\mathrm{SINR})$ on the fading might be approximately true when the SINR is not too high, cf [12, Remark 2.1]. Relations of this scheme to the Proportional Fair scheduling algorithm for the CDMA 1xEV-DO system are discussed in [12, Remark 2.2].

Example 4 (Truncated opportunistic channel scheduling): In practice, the scheduler gain is limited and the following variant of (5) is of interest

$$
g(n)= \begin{cases}1+1 / 2+\ldots+1 / n & \text { for } 1 \leq n \leq n_{\max } \\ g\left(n_{\max }\right) & \text { for } n>n_{\max }\end{cases}
$$

Numerical evidences show that the truncated opportunistic channel model is is a reasonable approximation of the opportunistic scheduler implemented in $4 \mathrm{G}$ networks; see Observation 1.

\section{B. Users and service policy}

We describe now the space-time process of user arrivals (and departures).

1) Traffic demand: As we already mentioned, we consider the VBR traffic: users arrive and request transitions of some data volumes. (The transmission rates vary in space and in time, depending on the user locations and the number of users served by the station at a given time; cf. Section II-A4.) More specifically, we assume a homogeneous time-space Poisson point process of user arrivals of intensity $\gamma$ arrivals per second per $\mathrm{km}^{2}$. This means that the time between two successive arrivals in a given zone of surface $S$ is exponentially distributed with parameter $\gamma \times S$, and all users arriving to this zone take their locations independently and uniformly. The time-space process of user arrivals is independently marked by random, identically distributed volumes of data the users want to download from their respective serving BS. These volumes are arbitrarily distributed and have mean $1 / \mu$ bits. Finally, we assume that the time-space point process of user arrivals marked by the data volumes is independent of the spatial, marked point process $\tilde{\Phi}$ of base stations.

The above arrival process induces the traffic demand per surface unit $\rho=\gamma / \mu$ expressed in bits per second per $\mathrm{km}^{2}$. Given $\tilde{\Phi}$, the traffic demand in the cell of any $\operatorname{BS} X \in \Phi$ equals

$$
\rho(X)=\rho|V(X)|,
$$

where $|A|$ denotes the surface of the set $A ; \rho(X)$ is expressed in bits per second.

2) Processor sharing systems: Given $\tilde{\Phi}$, the evolution of the number of users present in any given cell follows the (generalized) spatial, multi-class processor sharing queue model. That 
is, users start being served (downloading their volumes of data) immediately after their arrivals to cells. Their transmission rates depend on their SINR conditions and the number of users present in the same cell as described in Section II-A4. Users leave the system immediately after having downloaded their volumes. Note that given a realization of the point process $\Phi$ of base stations and its marks, the stochastic processes describing the evolution of different cells are independent.

\section{Generalized processor sharing queues}

Let us first recall some basic results regarding the steady state characteristics of the processor sharing queues describing the temporal evolution of the service in any given cell of the network. Then, we shall introduce a key concept of this paper that is cell load equations, allowing one to capture the spatial dependence of service processes in different cells.

1) Free cell performance characteristics: Given $\tilde{\Phi}$, for all $X \in \Phi$, denote by $\rho_{c}(X)$ the harmonic mean of the peak bit-rate over the cell $V(X)$

$$
\rho_{\mathrm{c}}(X):=\frac{|V(X)|}{\int_{V(X)} R^{-1}(\operatorname{SINR}(y, \tilde{\Phi})) d y},
$$

where $R^{-1}(\ldots)=1 / R(\ldots)$. We will call $\rho_{c}(X)$ the critical traffic demand of cell $X$. Together with the cell load $\rho(X)$ defined in (7) and the scheduling gain function $g(\cdot)$ it allows one to characterize the stability of the generalized processor sharing queue and express important mean characteristics of its steady state, and even characterize its distribution. Indeed, for any $X \in \Phi$ denote by $I(X), N(X)$, respectively, the probability that the cell $V(X)$ is idle (has no users) and the mean number of usersin this cell at the steady state. Also, denote by $r(x)$ the mean user throughput $r(X)$ in cell $V(X)$ defined as the ratio of the mean data volume to the mean typical user service time. Consider the following function $G(0):=1$ and for $n \geq 1, G(n):=g(1) \cdots g(n)$.

Proposition 2: [[47, 48]; cf. also [49, Proposition 3.1 and 3.2]] Assume that $g(n)>0$ for all $n \geq 1$ and that the limit in (9) exists and is strictly positive. Then, for any $X \in \Phi$, the processor sharing queue of cell $V(X)$ is stable if

$$
\rho(X)<\rho_{c}(X) \times \lim _{n \rightarrow \infty} g(n) .
$$

and unstable if $\rho(X)>\rho_{c}(X) \times \lim _{n \rightarrow \infty} g(n)$. Moreover, if (9) holds true then

$$
\begin{aligned}
I(X) & =\left(\sum_{n=0}^{\infty} \frac{\left(\rho(X) / \rho_{c}(X)\right)^{n}}{G(n)}\right)^{-1} \\
N(X) & =I(X) \sum_{n=1}^{\infty} n \frac{\left(\rho(X) / \rho_{c}(X)\right)^{n}}{G(n)} \\
r(X) & =\frac{\rho(X)}{N(X)}
\end{aligned}
$$

Denote by $\theta(X)$ the busy probability of cell $V(X)$

$$
\theta(X):=1-I(X)
$$

Note that under the assumptions of Proposition 2 if the stability condition (9) is satisfied then the infinite sum on the right-hand side of (10) converges, which is equivalent to $I(X)>0$, and to $\theta(X)<1$.
Example 5 (Round robin scheduling): In case $g(n) \equiv 1$, cf. Example 2, the cell is stable if $\rho(X)<\rho_{c}(X)$. Under this condition $\theta(X)=\rho(X) / \rho_{c}(X), N(X)=\theta(X) /(1-\theta(X))$ and $r(X)=\rho_{c}(X)-\rho(X)$.

Example 6 (Opportunistic scheduling): Applying the scheduling gain from Example 3 makes cells stable for all values of the traffic demand $\rho(X)<\infty$. The corresponding expressions for $\theta(X), N(X)$ and $r(X)$ are less explicit, but are still amenable to numerical calculations.

Remark 1: Observe that (numerically) inverting (10) in $\rho(X) / \rho_{c}(X)$, plugging it in into (11) and using (13) allow one to express the mean number of users $N(X)$ in terms of the cell load. This relation can be used to validate the generalized processor sharing model with some particular scheduling gain function $g(\cdot)$ with respect to real data. Numerical evidences presented in Section IV-B support the following statement.

Observation 1: The generalized processor sharing queue model with the considered truncated opportunistic scheduler (6) with $n_{\max }=100$ describes well the relation between the number of users and the cell load in the reference $4 G$ network described in Section IV-A1 for a large range of traffic conditions. The results are not sensitive to the choice of $n_{\max }$. The results are not sensitive to the choice of $n_{\max } \geq 20$.

2) Cell load equations and spatially correlated cells: The individual cell characteristics described in the previous section depend on the location of all base stations, shadowing realizations but also on the cell activity factors $\varphi_{Y}, Y \in \Phi$, introduced in Section II-A3 to account for the fact that BS might not transmit with their respective maximal powers $P_{Y}$.

It would be quite natural to assume that a given BS transmits with its maximal power $P_{Y}$ when it serves at least one user and does not transmit otherwise ${ }^{4}$. Taking this fact into account in an exact way would require defining $\varphi_{Y}$ as the indicators that $Y \in \Phi$ is busy, thus making $\varphi_{Y}$ dependent not only on $\tilde{\Phi}$ but also on the varying in time process of users. This, in consequence, would lead to the probabilistic dependence of the service processes at different cells, thus revoking the explicit expressions for their characteristics presented in Section II-C and, in fact, making the model analytically non-tractable. ${ }^{5}$

To avoid this difficulty we take into account the activity of station $Y$ in a simpler way, multiplying its maximal transmitted power by the probability $\theta(X)$ that it is busy (that is serves at least one user) in the steady state. In other words, in the SINR expression (3) we take $\varphi_{Y}=\theta(Y)$ where $\theta(Y)$ is the load of the cell $Y$; i.e.,

$$
\operatorname{SINR}(y, \tilde{\Phi})=\frac{1 / L_{X}(y)}{N+\sum_{Y \in \Phi \backslash\{X\}} \theta(Y) / L_{Y}(y)} .
$$

Making the above assumption, we preserve the independence of the queueing processes at all cells $V(X), X \in \Phi$, given the realization $\tilde{\Phi}$ of the process of BS with their shadowing and power marks. We call this simplifying assumption the timedecoupling of the cell processes. Note that the cell processes remain coupled in space. By this we mean two observations: Firstly, as in Section II-C1, $\rho(X)$ and $\rho_{c}(X)$ depend on

\footnotetext{
${ }^{4}$ Analysis of more sophisticated power control schemes is beyond the scope of this paper.

${ }^{5} \mathrm{We}$ are not aware of any result regarding the stability and performance of such a family of dependent queues.
} 
the realization of the process $\tilde{\Phi}$, in particular on the service zones $V(X)$ which are dependent sets. However, additionally, the critical traffic demands $\rho_{c}(X)$ of all stations $X \in \Phi$ (and in consequence all other time-stationary characteristics of the queueing processes) cannot be any longer calculated independently for all cells via (8), given $\tilde{\Phi}$, but are solutions of the following fixed point problem, which we call cell load equations: for all $X \in \Phi$

$$
\rho_{c}(X)=\frac{|V(X)|}{\int_{V(X)} R^{-1}\left(\frac{1 / L_{X}(y)}{N+\sum_{Y \in \Phi \backslash\{X\}} \theta(Y) / L_{Y}(y)}\right) d y},
$$

where $\theta(Y)$ depends on $\rho_{c}(Y)$ and the traffic demand $\rho(Y)$ via (13) and (10). This system of equations introduces additional spatial dependence between mean performance characteristics of different cells. It needs to be solved for $\left\{\rho_{c}(X)\right\}_{X \in \Phi}$ (equivalently for $\{\theta(X)\}_{X \in \Phi}$ ) given $\tilde{\Phi}$, i.e., network, power and shadowing realization. Other characteristics of each cell are then deduced from the cell load and traffic demand using the relations described in Section II-C, involving the scheduling gain function $g(\cdot)$.

We repeat, cell load equations (15) capture spatial dependence between the processor sharing queues of different cells, while allowing for their temporal independence, given the network realization.

Remark 2: [Network spatial stability] A natural question arises regarding the existence and uniqueness of the solution of the equations (15). Note that the mapping in the righthand-side of (15) is decreasing in all $\theta(Y), Y \in \Phi \backslash\{X\}$, provided the function $R$ is increasing, and thus, using (10) and (13), $\theta(X)$ is increasing in $\theta(Y), Y \in \Phi \backslash\{X\}$. Using this property it is easy to see that successive iterations of this mapping started either from $\theta(Y)=0$ for all $Y \in \Phi$ or from $\theta(Y)=1$ for all $Y \in \Phi$, converge to a minimal and maximal solution of (15), respectively. An interesting theoretical question regards the uniqueness of the solution of (15), in particular for a random, say Poisson, point process $\Phi$. Answering this question, which we call spatial stability of the model, is beyond the scope of this paper. Existence and uniqueness of the solution of a very similar problem (with finite number of stations and a discrete traffic demand) is proved in [24]. In the remaining part of the paper, to fix the attention, by the solution of (15) we understand the minimal one.

3) Equivalent form of cell load equations: In view of the future analysis, it is customary to consider for each station $X$ the ratio of the traffic demand to the critical traffic demand

$$
\rho^{\prime}(X):=\frac{\rho(X)}{\rho_{c}(X)} .
$$

We call $\rho^{\prime}(X)$ the normalized traffic demand. Note by (7) and (8)

$$
\rho^{\prime}(X)=\rho \int_{V(X)} R^{-1}(\operatorname{SINR}(y, \tilde{\Phi})) d y .
$$

All characteristics considered in Section II-C, including the stability condition, can be expressed in terms of the vector $\left(\rho(X), \rho^{\prime}(X)\right)$, with the following equivalent form of the cell load equations (15) with unknown vector $\left\{\rho^{\prime}(X)\right\}_{X \in \Phi}$ :

$$
\rho^{\prime}(X)=\rho \int_{V(X)} R^{-1}\left(\frac{1 / L_{X}(y)}{N+\sum_{Y \in \Phi \backslash\{X\}} \theta(Y) / L_{Y}(y)}\right) d y
$$

where

$$
\theta(Y)=1-\left(\sum_{n=0}^{\infty} \frac{\left(\rho^{\prime}(Y)\right)^{n}}{G(n)}\right)^{-1},
$$

with $G(\cdot)$ as in (10).

\section{SPATIAL NETWORK PERFORMANCE ANALYSIS}

Individual cell characteristics considered in Section II-C are important performance indicators of the service evaluated for each BS $X$ of the network. Mathematically, they form some non-independent marks of the points $X \in \Phi$, which are deterministic functions of $\tilde{\Phi}$ modeling locations of BS, their powers and the shadowing fields. If a given realization of the process $\tilde{\Phi}$ were an exact representation of some network, then the above characteristics would provide the QoS metrics in the respective cells. However, the point process $\tilde{\Phi}$ is usually merely a probabilistic model of the base station placement, and consequently a given point $X \in \Phi$ and its cell characteristics do not correspond to any really existing BS. Nevertheless, the whole family of cell characteristics, parametrized by $X \in \Phi$, does carry some information about the spatial distribution of the network performance characteristics. Consequently, when doing some appropriate averaging over the individual cell characteristics one can capture the global network performance laws. In what follows we propose two approaches in this regard, a detailed typical cell analysis and a simplified mean cell analysis.

\section{A. The detailed model and its typical cell analysis}

By the detailed model we understand the point process $\tilde{\Phi}$ with its individual cell characteristics considered in Section II-C as dependent marks. These marks are fully characterized by the two-dimensional mark $\left(\rho(X), \rho^{\prime}(X)\right)$, where the normalized traffic demands $\rho^{\prime}(X), X \in \Phi$ solve the (equivalent) cell load equations (18), cf. Section II-C3.

1) The typical cell of the detailed model: We are interested in the distribution of the vector $\left(\rho(0), \rho^{\prime}(0)\right)$ of the traffic demand and the normalized traffic demand of the typical station $^{6} X_{0}=0$ under the Palm distribution $\mathbf{P}^{0}$ of the stationary process $\tilde{\Phi}$. All individual cell characteristics of this typical station, considered in Section II-C, are deterministic functions of the vector $\left(\rho(0), \rho^{\prime}(0)\right)$,

Unfortunately, only the expected values $\mathbf{E}^{0}[\rho(0)]$ and $\mathbf{E}^{0}\left[\rho^{\prime}(0)\right]$ admit explicit expressions. Also, we have some variance results. These are key elements of some simplified analysis proposed in Section III-B.

\footnotetext{
${ }^{6}$ The typical cell of a stationary network is a mathematical formalization of a cell whose BS is "arbitrarily chosen" from the set of all stations, without any bias towards its characteristics. The formalization is made on the ground of Palm theory, where the typical cell $V(0)$ is this of the BS $X_{0}=0$ located at the origin under the Palm probability $\mathbf{P}^{0}$ associated to point process $\Phi$ and its stationary probability $\mathbf{P}$.
} 
2) Mean values: The vector $\left(\rho(0), \rho^{\prime}(0)\right)$ admits the following expressions regarding its (Palm) expectation.

Proposition 3: We have

$$
\begin{aligned}
\mathbf{E}^{0}[\rho(0)] & =\frac{\rho}{\lambda_{\mathrm{BS}}}, \\
\mathbf{E}^{0}\left[\rho^{\prime}(0)\right] & =\frac{\rho}{\lambda_{\mathrm{BS}}} \mathbf{E}[1 / R(\operatorname{SINR}(0, \tilde{\Phi}))] .
\end{aligned}
$$

The result (21) remains true for any stationary (translation invariant) assumption regarding the marks being the cell activity factors $\varphi_{Y}$, including the particular case $\varphi_{Y}=\theta(Y)$ being the solution of the cell load equations.

Proof: The first equation is quite intuitive: the average cell surface is equal to the inverse of the average number of BS per unit of surface. In the case of no shadowing $\mathbf{S}_{X}(y) \equiv 1$ the service zones $V(X)$ correspond to the Voronoi cells and both formulas can be derived directly from the classical inverse formula of Palm calculus [50, Theorem 4.2.1]. In general, they follow from a more general relation called typical location-station exchange formula in [20, Theorem 4.1.4]. It can be derived from the Campbell-Little-Mecke-Matthes formula interpreted as the mass transport principle between point process and Lebesgue measure, cf. [51, (10.3.1) and the proof of Theorem 11.2.1].

Remark 3: Note that the expectation in the right-hand-side of (21) is taken with respect to the stationary distribution of the BS process $\tilde{\Phi}$. It corresponds to the spatial average of the inverse of the peak bit-rate calculated throughout the network. The only random variable in this expression is the SINR experienced at an arbitrary location (chosen to be the origin 0) with respect to the base station serving this location (we denote this station $X_{*}$ in (23)) under the stationary distribution of the network $\tilde{\Phi}$. Due to the independence assumption of the process of user arrivals and $\tilde{\Phi}$, it is possible to identify this arbitrary location with the location of the typical user. Thus (21) can be called typical user-cell exchange formula.

Remark 4: [SINR distribution of the typical user] The distribution of $\operatorname{SINR}(0, \tilde{\Phi})$ is usually estimated in operational networks from user measurements. It also admits some analytic expressions for some particular point processes, in case of constant cell activity factors $\varphi_{Y}$ (without considering cell-load equations). The most studied is Poisson network model, where the distribution of the $\operatorname{SINR}$ at the typical location $\operatorname{SINR}(0, \tilde{\Phi})$ admits some explicit expressions; cf. [52-54]. Some expressions are also available for more regular than Poisson network models based on Ginibre and related determinantal point processes, cf. [55-57]. For a complete treatment of the SINR distribution of the typical user, see [20, Chapters 5-7].

3) Variance analysis: The following result is known for the Voronoi cells and was generalized to the service zones in the model with the shadowing.

Proposition 4: [[2]] The square relative standard deviations (SRSD) (with respect to Palm probability $\mathbf{P}^{0}$ of $\tilde{\Phi}$ )

$$
\frac{\operatorname{Var}^{0}(\rho(0))}{\left(\mathbf{E}^{0}[\rho(0)]\right)^{2}}
$$

of the traffic demand in the typical cell model is a scale free functional of the general network process $\tilde{\Phi}$ : it does not depend on the user traffic and is invariant to any homothetic transformation of the network process.
We were not able to prove similar result in full generality for the SRSD of the normalized traffic demand $\rho^{\prime}(0)$ in the typical cell. However, the following empirical observation was made and supported by numerical arguments in [2] in the case of round robin scheduling policy. We extend it to the model with the opportunistic scheduling.

Observation 2: In the Poisson network model specified in Section IV-A2, the SRSD of the normalized traffic demand and the logarithmic correlation coefficient of the typical cell under Palm probability $\mathbf{P}^{0}$ of $\tilde{\Phi}$

$$
\frac{\operatorname{Var}^{0}\left(\rho^{\prime}(0)\right)}{\left(\mathbf{E}^{0}\left[\rho^{\prime}(0)\right]\right)^{2}}, \quad \frac{\operatorname{Cov}^{0}\left(\ln \rho(0), \ln \rho_{c}(0)\right)}{\sqrt{\operatorname{Var}^{0}(\ln \rho(0)) \operatorname{Var}^{0}\left(\ln \rho_{c}(0)\right)}}
$$

do not depend significantly on $\rho$ (the traffic demand per surface unit). Moreover, traffic demand $\rho(0)$ and the critical traffic demand $\rho_{c}(0)$ are negatively correlated.

The numerical evidences are presented in Section IV-C. The negative correlation can be explained by the fact that $\rho(0)$ is proportional to the cell surface (18), while $\rho_{c}(0)$ is the harmonic average of the peak bit-rate function $R(\cdot)$ over the cell (17). For large cells this harmonic average is smaller due to small values of the peak bit-rate far from the base station.

4) Ergodic averaging in the detailed model: In order to evaluate further distributional characteristics of the random vector $\left(\rho(0), \rho^{\prime}(0)\right)$ one needs to approximate them by the spatial (empirical) averages of the vector $\left(\rho(X), \rho^{\prime}(X)\right)$ for $X \in \Phi$ considered (simulated) in a large enough window, leveraging the ergodicity of the point process $\tilde{\Phi} .{ }^{7}$ The following classical ergodic result is crucial in this regard.

Proposition 5: Consider an increasing network window A, say a disc (or square) centered at the origin and the radius (or side) increasing to infinity. If $\tilde{\Phi}$ is ergodic then

$$
\begin{aligned}
\mathbf{P}^{0} & \left\{\rho(0) \leq u, \rho^{\prime}(0) \leq t\right\} \\
& =\lim _{|A| \nearrow \infty} 1 / \Phi(A) \sum_{X \in A} \mathbf{1}\left(\rho(X) \leq u, \rho^{\prime}(X) \leq t\right) .
\end{aligned}
$$

The convergence is $\mathbf{P}$ almost sure.

Proof: The result follows from the ergodic theorem for point processes (see [50, Theorem 4.2.1], [45, Theorem 13.4.III]). More precisely, (22) follows straightforwardly from the ergodic result if the characteristics $\rho(X)$ and $\rho^{\prime}(X)$ for $X \in A$ are calculated taking into account the impact of the entire process $\tilde{\Phi}$ and not only other stations $Y \in A$. The proof of the convergence result in this latter case (more pertinent for practical applications) requires a careful study of the impact of the boundary effects, which is beyond the scope of this paper.

Remark 5: The following distributional characteristics of the typical cell, of particular interest for us, can be expressed as functionals $\mathbf{E}^{0}\left[f\left(\rho(0), \rho^{\prime}(0)\right]\right.$ and thus can be approximated by the empirical averages in the right-hand-side of (22) when taking large enough simulation window $A$.

- The mean traffic demand $\mathbf{E}^{0}[\rho(0)]$.

7 Note, when doing it, we are somehow coming back to the original intuition behind the mathematical concept of the typical point and cell. The difference is that the typical cell can be considered in a stationary non-ergodic setting, while the convergence of the spatial averages requires stronger, ergodic assumption. If the process is not ergodic however, then the interpretation of the mathematical concept of the typical cell is problematic. 
- The mean cell load $\mathbf{E}^{0}[\theta(0)]$ and the probability distribution function $\mathbf{P}^{0}\{\theta(0) \leq u\}$. The former gives the average load of the network, while the later gives the fraction of cells with load below some value $u<1$ considered as a critical one. ${ }^{8}$

- The mean $\mathbf{E}^{0}[N(0)]$ and the median $m$ of the number of users (defined by $\mathbf{P}^{0}\{N(0) \leq m\}=1 / 2$ ) in the typical cell. The median is more appropriate for networks with large disparity of cell sizes, where a few heavily loaded cells can significantly bias the mean $\mathbf{E}^{0}[N(0)]$. $^{9}$

- The mean user throughput $\mathbf{E}^{0}[r(0)]=\mathbf{E}^{0}[\rho(0) / N(0)]$. Note this is a double averaging: $\rho(0) / N(0)$ corresponds to the time-average user throughput in the typical cell and the palm expectation $\mathbf{E}^{0}$ corresponds to the spatial average of this quantity over the network cells. Note, in general $\mathbf{E}^{0}[r(0)] \neq \mathbf{E}^{0}[\rho(0)] / \mathbf{E}^{0}[N(0)] .^{10}$

\section{B. Gaussian approximations}

The expectations given in Proposition 3 do not give enough information regarding the distribution of the vector $\left(\rho(0), \rho^{\prime}(0)\right)$. For example, they do not allow one to calculate $\mathbf{P}^{0}\{\theta(0) \leq u\}, \mathbf{E}^{0}[N(0)]$ and $\mathbf{E}^{0}[r(0)]=\mathbf{E}^{0}[\rho(0) / N(0)]$ even in the round robin case. ${ }^{11}$ It is only through the empirical averaging of the characteristics of many cells that we can approximate them in the detailed model. Also, the evaluation of this detailed model requires solving of the system of load equations (18) for many cells. In this section we propose an alternative approach, where the vector $\left(\rho(0), \rho^{\prime}(0)\right)$ will be approximated by some two dimensional log-normal vector. Mean and variance analysis of the typical cell, presented in Sections III-A2 and III-A3 is crucial in this regard.

1) Mean cell load equation: We propose first a simplified way of capturing the dependence of the cell loads. The motivation comes from (21), which we write here in a detailed way, with the explicit dependence of the $\operatorname{SINR}(0, \tilde{\Phi})$ on the cell loads, as in (18)

$\mathbf{E}^{0}\left[\rho^{\prime}(0)\right]=\frac{\rho}{\lambda_{\mathrm{BS}}} \mathbf{E}\left[R^{-1}\left(\frac{1 / L_{X_{*}}(0)}{N+\sum_{Y \in \Phi \backslash\left\{X_{*}\right\}} \theta(Y) / L_{Y}(0)}\right)\right]$

Recall that $\theta(Y)$ depends on $\rho^{\prime}(Y)$ via (19) and that in order to evaluate the right-hand-side of (23) one needs to solve the detailed system of the cell load equations (18), which is computationally quite a heavy task, especially if many stations are simulated. To simplify this part of the model evaluation, as an alternative approach, called the mean cell approach, we propose to replace (23), where $\theta(Y)$ for all $Y \in \Phi$ are

\footnotetext{
${ }^{8}$ Note that the opportunistic scheduling considered in Examples 6 and 3 makes all cells are stable, $\mathbf{P}^{0}\{\theta(0)<1\}=1$. For the round robin scheme, in the infinite Poisson network model (corresponding to a very large irregular network, exhibiting arbitrarily large cells) $\mathbf{P}^{0}\{\theta(0)<1\}<1$, for all values of the traffic demand $\rho>0$, i.e., there is a fraction of unstable cells, even for an arbitrarily small values of the traffic demand per surface unit.

${ }^{9}$ This artifact is best seen in Poisson model with round robin scheme where $\mathbf{E}^{0}[N(0)]=\infty$, for all $\rho>0$, cf. Footnote 8 .

${ }^{10} \mathbf{E}^{0}[\rho(0)] / \mathbf{E}^{0}[N(0)]$ corresponds to the ratio of the spatial averages of the traffic demand and the number of users. It has a drawback of being seriously biased by a few heavily loaded cells. In Poisson model with round robin scheme it is equal to 0 for all $\rho>0$, since $\mathbf{E}^{0}[N(0)]=\infty$.

${ }^{11}$ In the case of round robin $\theta(X)=\rho^{\prime}(X)$.
}

calculated via (19) and the system of equations (18), by the following two equations in two unknown constants $\bar{\rho}^{\prime}$ and $\bar{\theta}$

$$
\begin{aligned}
\bar{\rho}^{\prime} & =\frac{\rho}{\lambda_{\mathrm{BS}}} \mathbf{E}\left[R^{-1}\left(\frac{1 / L_{X_{*}}(0)}{N+\sum_{Y \in \Phi \backslash\left\{X_{*}\right\}} \bar{\theta} / L_{Y}(0)}\right)\right] \\
\bar{\theta} & =1-\left(\sum_{n=0}^{\infty} \frac{\left(\bar{\rho}^{\prime}\right)^{n}}{G(n)}\right)^{-1}
\end{aligned}
$$

where (24) and (25) mimic, respectively, (23) and (19). We have the following result regarding the existence of solutions. Uniqueness has to be conjectured for given functions $R(\cdot)$, $G(\cdot)$ and the distribution of the point process $\tilde{\Phi}$. In the remaining part of the paper, to fix the attention, by the solution of the system of equations (24) and (25) we understand the solution with minimal $\bar{\theta}$.

Proposition 6: If $R(\cdot)$ is a non-decreasing function and $\bar{\theta}\left(\bar{\rho}^{\prime}(\bar{\theta})\right)$ is a continuous function of $\bar{\theta} \in[0,1]$ then the system of equations (24) and (25) has at least one solution with $\bar{\theta}$ in $[0,1]$.

Proof: It is easy to see that if $R(\cdot)$ is a non-decreasing function then $\bar{\rho}^{\prime}(\bar{\theta})$, defined as the right-hand-side of $(24)$, is non-decreasing in $\bar{\theta}$. Also $\bar{\theta}\left(\bar{\rho}^{\prime}\right)$, the right-hand side of (25), is a non-decreasing function of $\bar{\rho}^{\prime}$. Thus the composition $f(\bar{\theta}):=$ $\bar{\theta}\left(\bar{\rho}^{\prime}(\bar{\theta})\right)$ is non-decreasing, and satisfies $f(0) \geq 0$ and $f(1) \leq$ 1. Consequently the equation $\bar{\theta}=f(\bar{\theta})$ (and hence the system of equations (24) and (25)) has at least one solution with $\bar{\theta}$ in $[0,1]$ provided $f(\bar{\theta})$ is continuous.

In practice, the evaluation of the right-hand-side of (24) still requires simulations of the detailed model $\tilde{\Phi}$ to perform empirical averaging of $R^{-1}(\operatorname{SINR}(y, \tilde{\Phi})$ over a large number of locations $y$ in the simulation window (and possibly for several realizations of the detailed model, cf Section IV-A3) as no closed form expression is known even in the case of Poisson network. ${ }^{12}$ ) However, there is no need to solve the detailed cell load equations.

2) Gaussian approximate model: In this simplified model, the distribution of the vector $\left(\rho(0), \rho^{\prime}(0)\right)$, considered under palm probability $\mathbf{P}^{0}$ of $\tilde{\Phi}$, is approximated by the distribution of some jointly log-normal vector $\left(\rho_{0}, \rho_{0}^{\prime}\right)$, whose parameters will be specified via $\bar{\rho}, \bar{\rho}^{\prime}$ and some characteristics of the network model $\tilde{\Phi}$, which do not depend on the user traffic.

More specifically, we consider the following representation

$$
\rho_{0}=e^{\mu+\sigma N}, \quad \rho_{0}^{\prime}=e^{\mu^{\prime}+\sigma^{\prime} N^{\prime}},
$$

where $\left(N, N^{\prime}\right)$ is a vector of the standard, jointly Gaussian variables, which are in general not independent. The parameters of the representation (26) are related to the means $\bar{\rho}:=\mathbf{E}\left[\rho_{0}\right]$ and $\bar{\rho}^{\prime}:=\mathbf{E}\left[\rho_{0}^{\prime}\right]$ and variances $v:=\operatorname{Var}\left(\rho_{0}\right)$, $v^{\prime}:=\operatorname{Var}\left(\rho_{0}^{\prime}\right)$ of $\left(\rho_{0}, \rho_{0}^{\prime}\right)$ in the following way $\mu=2 \ln \bar{\rho}-$ $\frac{1}{2} \ln \left(v+\bar{\rho}^{2}\right), \sigma^{2}=\ln \left(v+\bar{\rho}^{2}\right)-2 \ln \bar{\rho}$ and similarly for $\mu^{\prime}, \sigma^{\prime}$. We further use the SRSD $\alpha:=\operatorname{Var}\left(\rho_{0}\right) / \bar{\rho}^{2}$ and $\alpha^{\prime}:=\operatorname{Var}\left(\rho_{0}^{\prime}\right) / \bar{\rho}^{\prime 2}$ to represent variances $v$ and $v^{\prime}$, in terms

\footnotetext{
${ }^{12}$ An alternative approach to obtain $\mathbf{E}\left[R^{-1}(\operatorname{SINR}(0, \tilde{\Phi}))\right]$ consists in the numerical integration with respect to the distribution of the SINR of the typical user, modified to allow for a constant $\bar{\theta}$ weighting the interference term, which admits some more explicit expressions for some network models, cf. Remark 4.
} 
of the means $\bar{\rho}$ and $\bar{\rho}^{\prime}$. Summarizing

$$
\begin{aligned}
\mu & =2 \ln \bar{\rho}-\frac{1}{2} \ln \left(\left(\bar{\rho}^{2}(\alpha+1)\right),\right. \\
\sigma^{2} & =\ln \left(\bar{\rho}^{2}(\alpha+1)\right)-2 \ln \bar{\rho}
\end{aligned}
$$

and similarly for $\mu^{\prime}, \sigma^{\prime}$.

The dependence between $N$ and $N^{\prime}$ is specified via the logarithmic correlation coefficient $c$ of $\rho_{0}$ and $\rho_{0} / \rho_{0}^{\prime}$.

$$
c:=\frac{\operatorname{Cov}\left(\ln \rho_{0}, \ln \left(\rho_{0} / \rho_{0}^{\prime}\right)\right)}{\sqrt{\operatorname{Var}\left(\ln \rho_{0}\right) \operatorname{Var}\left(\ln \left(\rho_{0} / \rho_{0}^{\prime}\right)\right)}} .
$$

Note that if $\left(\rho_{0}, \rho_{0}^{\prime}\right)$ approximate $\left(\rho(0), \rho^{\prime}(0)\right)$ of the typical cell, then $\rho_{0} / \rho_{0}^{\prime}$ approximates $\rho_{c}(0)$. The following relations hold between $c$ and $C:=\operatorname{Cov}\left(N, N^{\prime}\right)=E\left[N N^{\prime}\right]$.

Proposition 7: We have

$$
\begin{aligned}
c & =\frac{\sigma-\sigma^{\prime} C}{\sqrt{\sigma^{2}+{\sigma^{\prime}}^{2}-2 \sigma \sigma^{\prime} C}}, \\
C & =\frac{1}{\sigma^{\prime}}\left(\sigma\left(1-c^{2}\right)-c \sqrt{\left(c^{2}-1\right) \sigma^{2}+{\sigma^{\prime}}^{2}}\right) .
\end{aligned}
$$

Proof: The expression in (29) can be derived directly from the definition of $c$. In order to prove (??) we use the following equivalent representation of the distribution of the vector $\left(\rho_{0}, \rho_{0}^{\prime}\right)$ proposed in [2]: Assume a vector $\left(Z, Z_{c}\right)$ of independent, standard Gaussian variables and take $\rho_{0} / \rho_{0}^{\prime}:=e^{\mu_{c}+\sigma_{c} Z_{c}}$, $\rho_{0}:=e^{\mu+c \sigma Z_{c}+\sigma \sqrt{1-c^{2}} Z}, \quad \rho_{0}^{\prime}:=e^{\mu^{\prime}+\left(c \sigma-\sigma_{c}\right) Z_{c}+\sigma \sqrt{1-c^{2}} Z}$, where $\mu_{c}:=\mu-\mu^{\prime}$ and $\sigma_{c}:=c \sigma+\sqrt{\left(c^{2}-1\right) \sigma^{2}+\sigma^{\prime 2}}$. Equating the logarithmic standard deviation of $\rho_{0} / \rho_{0}^{\prime}$ obtained with two representations one obtains the relation

$$
\sqrt{\sigma^{2}+{\sigma^{\prime}}^{2}-2 \sigma \sigma^{\prime} C}=c \sigma+\sqrt{\left(c^{2}-1\right) \sigma^{2}+{\sigma^{\prime}}^{2}}
$$

which can be solved in $C$ thus giving (30).

The distribution of $\left(\rho_{0}, \rho_{0}^{\prime}\right)$ is thus completely determined by the parameters $\bar{\rho}, \bar{\rho}^{\prime}, \alpha, \alpha^{\prime}, c$, which we fix as follows.

a) Mean specification: We assume the value of $\bar{\rho}$ as in (20) (that is equal to $\mathbf{E}^{0}[\rho(0)]$ ) and the value of $\bar{\rho}^{\prime}$ as the solution of the (simplified) mean cell load equation (24) with (25).

b) Variance and covariance specification: Following Proposition 4 and Observation 2 we take

$$
\begin{aligned}
\alpha & =\frac{\operatorname{Var}^{0}(\rho(0))}{\left(\mathbf{E}^{0}[\rho(0)]\right)^{2}}, \\
\alpha^{\prime} & \approx \frac{\operatorname{Var}^{0}\left(\rho^{\prime}(0)\right)}{\left(\mathbf{E}^{0}\left[\rho^{\prime}(0)\right]\right)^{2}}, \\
c & \approx \frac{\operatorname{Cov}^{0}\left(\ln \rho(0), \ln \rho_{c}(0)\right)}{\sqrt{\operatorname{Var}^{0}(\ln \rho(0)) \operatorname{Var}^{0}\left(\ln \rho_{c}(0)\right)}} .
\end{aligned}
$$

Proposition 4 says that $\alpha$ theoretically does not depend on the traffic parameter $\rho$ (and even on the homothetic scaling of the network $\tilde{\Phi}$ ). Observation 2 says that $\alpha^{\prime}$ and $c$ can be in practice estimated on $\tilde{\Phi}$ via the spatial averaging (22) and appropriate log-linear regressions, regardless of the traffic parameter $\rho$. .

The log-normal vector $\left(\rho_{0}, \rho_{0}^{\prime}\right)$ is called the Gaussian approximate model. Numerical evidences supporting the following statement are presented in Section IV-D.

Observation 3: Consider Poisson network model specified in Section IV-A2 and the opportunistic scheduler (6) with $n_{\max }=$
100. The distribution of the vector $\left(\rho_{0}, \rho_{0}^{\prime}\right)$ in the Gaussian approximate model with parameters (34) well approximates the Palm distribution of $\left(\rho(0), \rho^{\prime}(0)\right)$ in the detailed model with respect to the characteristics $f\left(\rho(0), \rho^{\prime}(0)\right)$ of the typical cell listed in Remark 5

$$
\mathbf{E}^{0}\left[f\left(\rho(0), \rho^{\prime}(0)\right)\right] \approx \mathbf{E}\left[f\left(e^{\mu+\sigma N}, e^{\mu^{\prime}+\sigma^{\prime} N^{\prime}}\right)\right] .
$$

Note that $\mathbf{E}\left[f\left(e^{\mu+\sigma N}, e^{\mu^{\prime}+\sigma^{\prime} N^{\prime}}\right)\right]$ can be (in general) evaluated by numerical integration using the two-dimensional Gaussian distribution of $\left(N, N^{\prime}\right)$ with the specified mean and covariance matrix. Recall, that this requires: estimation from $\tilde{\Phi}$ of the coefficients $\alpha, \alpha^{\prime}, c$ which do not depend on the traffic demand, solution of the equation (24), for $\bar{\rho}^{\prime}$ (together with (25)), which in practice, requires simulations of $\tilde{\Phi}$ to evaluate the expectation in the right-hand-side of (24), and calculating the simple expression (20) for $\bar{\rho}$, which can be also considered as the input (traffic) parameter of the model. Parameters $\bar{\rho}$ and $\bar{\rho}^{\prime}$ are the only model variables depending on the traffic demand per surface $\rho$.

Finally, we formulate the following observation regarding the Gaussian approximate model based on real data comparative study presented in Section IV-E.

Observation 4: The distribution of the mean cell load, mean number of users and the user throughput calculated using Gaussian approximate model as in Observation 3 exhibits a relatively good agreement with the real data corresponding to the reference $4 G$ network described in Section IV-AI.

\section{NUMERICAL ANALYSIS}

The results presented in this section are organized as follows. First we validate the usage of the generalized processor sharing model with respect to the real data from some reference operational network, thus supporting Observation 1. This does not require any spatial network model.

Then we place this processor sharing model within our spatial network model and perform the spatial network analysis described in Section III. This includes the estimation of the parameters of the Gaussian approximate model of (with the justification of Observation 2), and validation of this model with respect to the detailed typical cell analysis based on the ergodic averaging (Observation 3) as well as with respect to the real data from the reference operational network (Observation 4). Then, we use the validated Gaussian approximate model to make some performance prediction for the reference operational network.

We begin with the description of the operational network and its stochastic model.

\section{A. Reference operational network and its Poisson model}

We consider a large area representative of some European country comprising a mix of urban, suburban and rural zones with $4 \mathrm{G}$ network deployed. ${ }^{13}$

\footnotetext{
${ }^{13}$ Spatial heterogeneity of a cellular network (existence of urban, suburban and rural zones) is taken into account in a way proposed in [43]. This approach relies on an observation that the distance coefficient in the propagation loss function depends on the type of zone in such a way that its product to the distance between neighboring base stations remains approximately constant In this case the relations of the mean cell load, number of users and users throughput to mean traffic demand per cell, are the same for the different types of zones.
} 
1) $4 G$ network: The carrier frequency $f_{0}=2.6 \mathrm{GHz}$, with a frequency bandwidth $W=20 \mathrm{MHz}$, base station power $P=63 \mathrm{dBm}$ and noise power $N=-90 \mathrm{dBm}$. The function $R(\cdot)$ expressing the peak bit-rate in relation to the SNR is estimated to be $R(\xi)=b R_{\mathrm{MIMO}}(\xi)$, where $R_{\mathrm{MIMO}}(\cdot)$ is the theoretical rate of $2 \times 2$ MIMO given by (4) and the calibration coefficient $b=0.35$. Each BS comprises three antennas having each a three-dimensional radiation pattern specified in [3, Table A.2.1.1-2].

The real-life measurements are collected from the reference network using a tool which is used by operational engineers for network maintenance. This tool measures several parameters for every base station and for each hour during a day. This allows us to know the traffic demand, cell load and users number for each cell in each hour.

2) Poisson network model: Knowing the BS coordinates and the surface of the deployment zone we deduce the BS density $\lambda_{\mathrm{BS}}=1.27$ stations per $\mathrm{km}^{2}$ for the typical urban network zone.

For the analytic model, the locations of BS is modelled by a homogeneous Poisson point process with intensity $\lambda$. It is marked by constant transmission powers $P_{n}=P=$ $63 \mathrm{dBm}$ and independent log-normal shadowing fields $\mathbf{S}_{n}(\cdot)$, assumed moreover uncorrelated (across receiver location) with marginals having unit mean and logarithmic standard deviation $\sigma_{S}=12 \mathrm{~dB}$. We assume the distance-loss function $l(r)=(K r)^{\beta}$ with the propagation exponent $\beta=3.88$ and the constant $K=3176 \mathrm{~km}^{-1}$.

The BS and mobile antenna heights are assumed to be equal to $30 \mathrm{~m}$ and $1.5 \mathrm{~m}$ respectively. The common channels power is taken equal to $10 \%$ of the total base station power. The handover margin is taken $6 \mathrm{~dB} .{ }^{14}$

3) Model numerical evaluation methodology: When studying this model through the detailed typical cell analysis, the mean traffic demand $\rho$ per surface unit is used as the input of the typical cell model. The performance metrics of the model are estimated by the simulation of 10 realizations of the Poisson model with (roughly) 300 cells for each traffic demand $\rho$. For each realization, we compute the individual cell characteristics solving the cell load equations $(18)^{15}$ We use and calculate the spatial distribution of these characteristics using the ergodic approach from Section III-A4. The same realizations are used to estimate the fixed parameters $\alpha, \alpha^{\prime}, c$ of the Gaussian approximate model, cf. Section III-B2. Other realizations of the Poisson network model are used to estimate parameters (24)-(25) of the Gaussian approximate model for different values of the traffic demand per surface. This, together with the simple expression for $\bar{\rho}$, allows us to specify the distribution of the fundamental vector $\left(\rho_{0}, \rho_{0}^{\prime}\right)$ of this simplified model and use it to evaluate the approximations of all considered performance characteristics.

\footnotetext{
${ }^{14}$ Mobile users connect to the base station randomly uniformly chosen among stations offering received power within the margin of $6 \mathrm{~dB}$ with respect to the strongest station.

${ }^{15}$ Solving 300 cell load equations takes roughly one hour, when using Matlab function fsolve on a standard PC laptop.
}
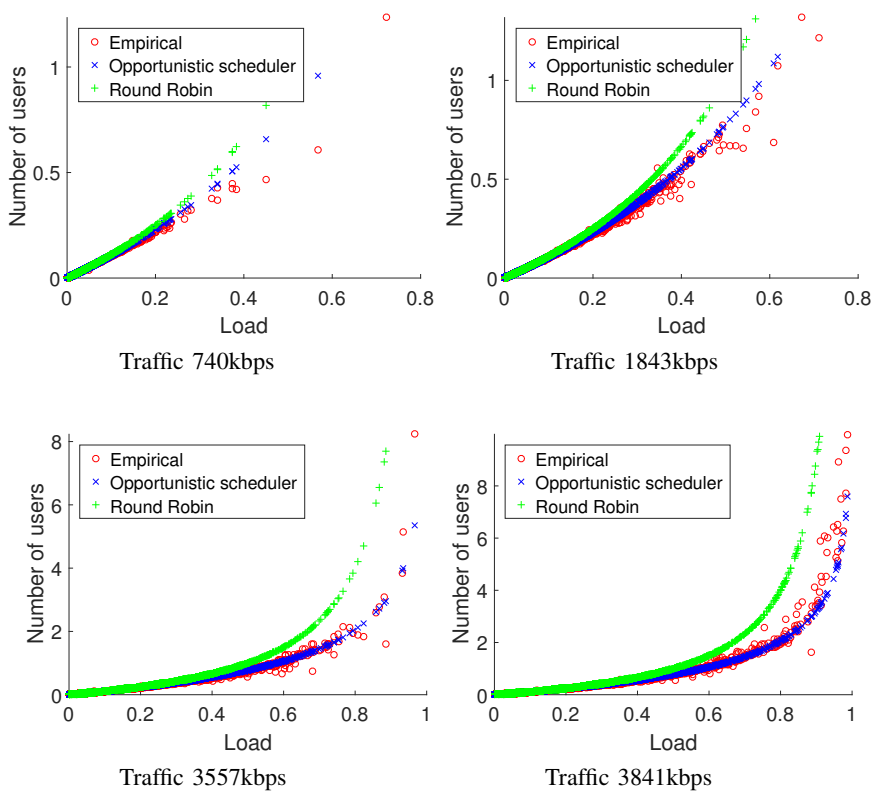

Fig. 1: The relation between the number of users in the cell to the cell load for various values of the mean cell traffic demand $\bar{\rho}$; empirical data, round-robin and truncated opportunistic model.

\section{B. Validation of the opportunistic scheduler model}

In this section we present numerical evidences supporting Observation 1. In this regard, we collect the data from our reference $4 \mathrm{G}$ network for some given day; different hours correspond to different mean traffic demand $\bar{\rho}$ per cell. For a given hour, we obtain the number of users and the cell load (fraction of the hour the cell was serving at least one user) for all cells of the reference network. We plot these points on Figure 1 and compare to the analytic curves obtained from the generalized processor sharing queue model with the truncated opportunistic scheduler of Example 4 with $n_{\max }=100$, as explained in Remark 1. For comparison, we plot also the analogue value of $N(X)$ obtained under the assumption of the round robin scheduler. Figure 1 shows that the generalized processor sharing queue model with the considered truncated opportunistic scheduler describes well the relation between the number of users and the cell load in the considered reference network under different traffic conditions. The results are not sensitive to the choice $n_{\max }=100$ with very similar results obtained for $n_{\max } \geq 20$. This brings numerical evidences supporting the statement in Observation 1.

\section{Estimation of the fixed parameters of the Gaussian approx- imate model}

In order to specify the Gaussian approximate model described in Section III-B2 and validate Observation 2, we assume the truncated opportunistic scheduler validated in Section IV-B, we place it in the context of our Poisson network model and estimate the parameters $\alpha, \alpha^{\prime}, c$ in (31), (32) and (33).

Figures 2(a) and (b) show the linear regression curves for the SRSD parameters $\alpha$ and $\alpha^{\prime}$ of the traffic demand and the normalized traffic demand, respectively. They bring numerical evidences supporting Proposition 4 and validate the first part of Observation 2. The second part of Observation 2, regarding 


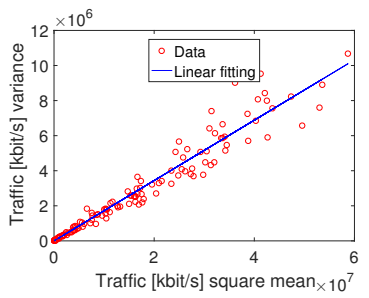

(a) Linear regression for $\alpha$

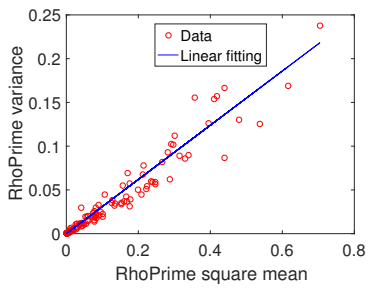

(b) Linear regression for $\alpha^{\prime}$

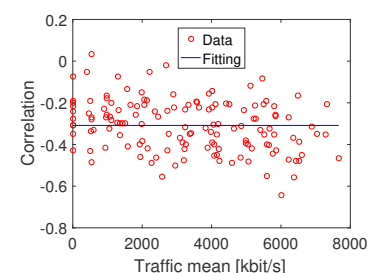

(c) Logarithmic correlation coefficient $c$

Fig. 2: Linear regression estimation of the SRSD coefficientsw $\alpha$ and $\alpha^{\prime}$ on Figures (a) and (b), and the logarithmic correlation coefficient $c$ on Figure (c), based on different values of the mean traffic demand per cell in the simulated typical cell model.

the invariance with respect to the traffic of the logarithmic correlation coefficient between the traffic demand and the critical traffic demand is supported by the results presented on Figure 2(c). We infer from this study the following estimated values of these parameters:

$$
\alpha=0.172057, \quad \alpha^{\prime}=0.309799, \quad c=-0.308575 .
$$

Note that $c<0$ meaning that the traffic demand $\rho(0)$ and the critical traffic demand $\rho_{c}(0)$ are negatively correlated.

\section{Gaussian approximate vs detailed model}

In this section we extensively validate the Gaussian approximate model from Section III-B2 with respect to the detailed typical cell model thus bringing numerical evidences supporting the statement in Observation 3. We assume the parameters estimated in Section IV-C and study the cumulative distribution function (CDF) of several cell characteristics for the typical cell and its Gaussian approximate mean cell approximation. Figures 5, 6, 7, 8, 9, 10 (which are presented at the end of the paper) show the spatial CDF of the critical traffic demand, normalized traffic demand, cell load, mean number of users and the user throughput, respectively, for different traffic regimes. The term "Empirical" and "Analytic" used in the legends of these figures correspond to the CDF estimated by the ergodic spatial averaging over the cells of the detailed model and the calculations made using the Gaussian approximate model, respectively.

We conclude that the Gaussian approximate model is a good approximation of the typical cell model. It will be our main tool in the analysis of the real reference network.

\section{E. Gaussian approximate model cell vs real data}

In this section we validate the Gaussian approximate model with respect to the real data from the reference operational network providing numerical evidences for Observation 4. Figure 3 shows different characteristics (mean or median, standard deviation, as well as 5 and 95-percentiles) of the spatial distribution of the cell performance metrics considered in this paper. On these figures the term "Empirical" corresponds to
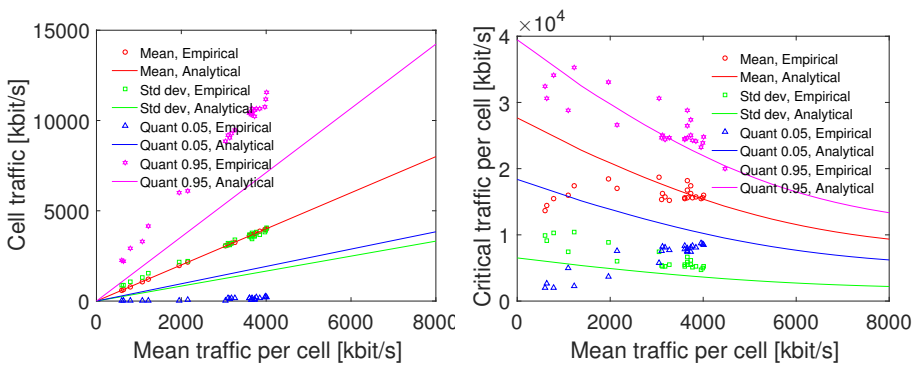

Traffic demand

Critical traffic demand

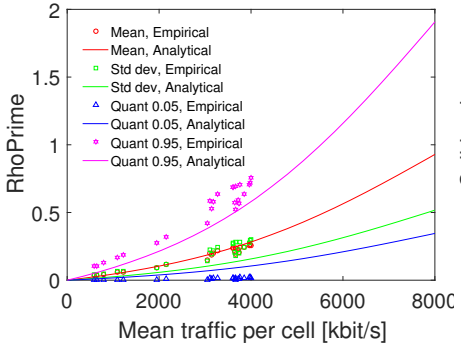

Normalized traffic demand

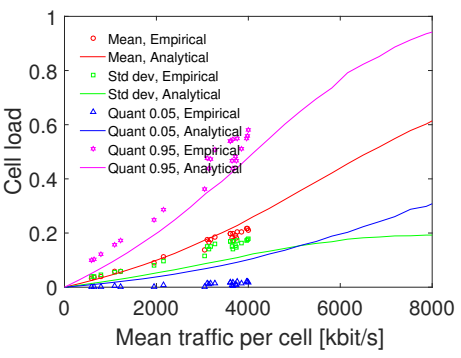

Cell load

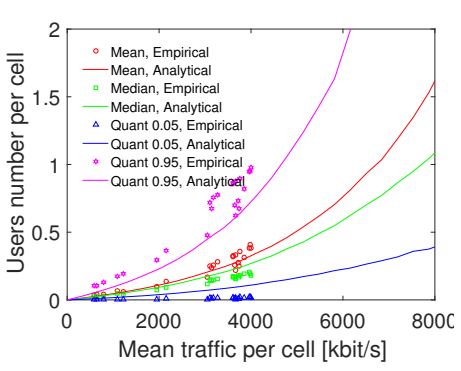

Number of users

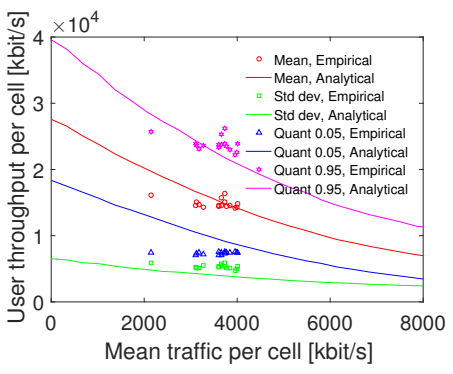

User throughput

Fig. 3: Study of the spatial distribution of cell characteristics. Real data and the Gaussian approximate model.

the real data collected in the reference network. The following observations can be made.

1) Spatial averages: We observe a good matching of the studied spatial mean and median cell characteristics.

2) Spatial variability: We observe that the traffic demand per cell exhibits in the reference network more spatial variability (larger variance, bigger 95-percentiles and smaller 5-percentiles) than predicted by our Gaussian approximate model. Assuming that the real reference network does not exhibit more spatial variability regarding the cell sizes than our Poisson network model, the above observation might suggest that the real traffic demand process is not spatially homogeneous. As a consequence the spatial variability of the critical traffic demand, normalized traffic demand and the cell load calculated in the reference network is also bigger than predicted by the model. However, the differences between the empirical and analytic values of the variances and quantiles are in general much smaller than in the case of the traffic demand, thus showing a relatively good agreement between the model and the real data.

\section{F. Throughput prediction}

In this final numerical exercise we present on Figure 4 the prediction of the evolution of the mean user throughput in the 


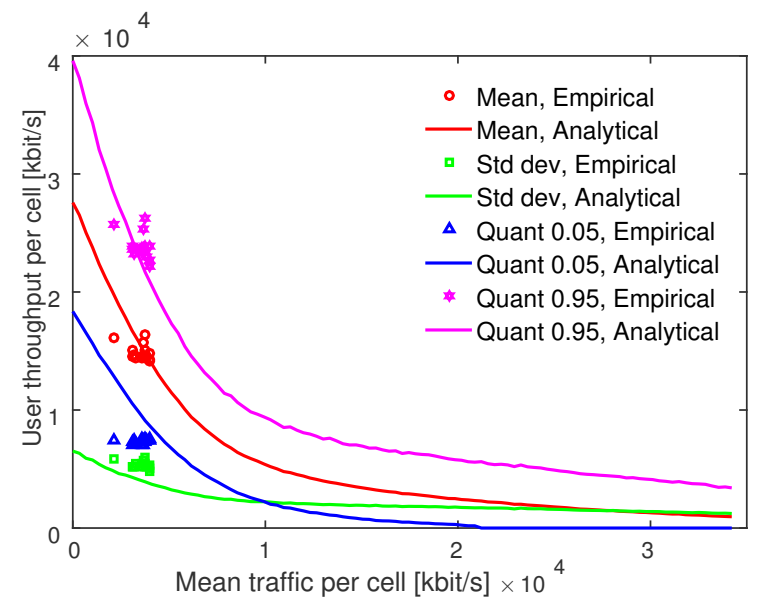

Fig. 4: Prediction of the evolution of the mean user throughout.

reference network well beyond the currently observed traffic demand (corresponding to "Empirical" points on the presented figure). The possibility to make such a prediction for various frequency spectra, density of base stations, scheduler, and linklayer technology (MIMO 2x2, 3x2 etc) is crucial for network operators for the strategic dimensioning planning. It allows them to estimate the required resources (and compare the costs of these alternative solutions) to ensure that a sufficiently large fraction of the network (typically 95\%) offers to user the targeted throughput.

\section{CONCLUSION}

We have presented a comprehensive framework allowing cellular network operators to evaluate the performance of their networks and helping them to take strategic decisions regarding network dimensioning. It consists in a synthetic mathematical model based on information theory, queueing theory and stochastic geometry, whose elements can be configured to represent various layers of the given wireless cellular network. Evaluation of the model requires only static simulations to estimate some stochastic geometric expectations which makes the proposed approach significantly more rapid than pure simulations often used by network operators for this purpose. We provide also a methodology allowing operators to compare and keep the model adequate to the real data they systematically collect in their networks, thus increasing the reliability of the network performance predictions. Awaiting for more massive deployment of $5 \mathrm{G}$ networks to get more representative data, in an ongoing work we extend our framework to the analysis of this new wireless network technology.

Acknowledgment: The authors would like to thank Mengting XIA for her careful reading of the manuscript and for making various corrections and suggestions.

\section{REFERENCES}

[1] B. Błaszczyszyn, M. Jovanovic, and M. K. Karray, "How user throughput depends on the traffic demand in large cellular networks," in Proc of WiOpt/SpaSWiN, 2014.

[2] B. Błaszczyszyn, R. Ibrahim, and M. Karray, "Spatial disparity of QoS metrics between base stations in wireless cellular networks," IEEE Trans. Commun., vol. 64, no. 10, p. 4381, 2016, publised online 16 August 2016.
[3] 3GPP, "TR 36.814-V900 Further advancements for E-UTRA Physical Layer Aspects," in 3GPP Ftp Server, 2010.

[4] G. Piro, L. A. Grieco, G. Boggia, F. Capozzi, and P. Camarda, "Simulating LTE cellular systems: an open source framework," IEEE Trans. Veh. Technol., vol. 60, pp. 498-513, 2011.

[5] C. Mehlführer, J. C. Ikuno, M. Simko, S. Schwarz, M. Wrulich, and M. Rupp, "The Vienna LTE simulators - enabling reproducibility in wireless communications research," EURASIP Journal on Advances in Signal Processing, vol. 29, pp. 1-14, 2011.

[6] M. Simko, Q. Wang, and M. Rupp, "Optimal pilot symbol power allocation under time-variant channels," EURASIP Journal on Wireless Communications and Networking, vol. 2012:225, no. 25, pp. 1-11, 2012.

[7] N. Baldo, M. Miozzo, M. Requena, and J. N. Guerrero, "An open source product-oriented LTE network simulator based on ns-3," in Proc. of MSWIM, 2011.

[8] N. Baldo, M. Requena, J. Nin, and M. Miozzo, "A new model for the simulation of the LTE-EPC data plane," in Proc. of WNS3, 2012.

[9] A. J. Goldsmith and S.-G. Chua, "Variable-rate variable-power MQAM for fading channels," IEEE Trans. Commun., vol. 45, pp. 1218-1230, 1997.

[10] P. E. Mogensen, W. Na, I. Z. Kovács, F. Frederiksen, A. Pokhariyal, K. I. Pedersen, T. E. Kolding, K. Hugl, and M. Kuusela, "LTE capacity compared to the Shannon bound," in Proc. of VTC Spring, 2007, pp. 1234-1238.

[11] M. K. Karray, M. Jovanovic, and B. Błaszczyszyn, "Theoretical expression of link performance in OFDM cellular networks with MIMO compared to simulation and measurements," ANN TELECOMMUN, vol. 70, no. 11-12, pp. 479-490, 2015.

[12] S. Borst, "User-level performance of channel-aware scheduling algorithms in wireless data networks," in Proc. of IEEE INFOCOM, Mar. 2003.

[13] T. Bonald and A. Proutière, "Wireless downlink data channels: user performance and cell dimensioning," in Proc. of Mobicom, Sep. 2003.

[14] N. Hegde and E. Altman, "Capacity of multiservice WCDMA networks with variable GoS," in Proc. of IEEE WCNC, 2003.

[15] T. Bonald, S. C. Borst, N. Hegde, M. Jonckheere, and A. Proutière, "Flow-level performance and capacity of wireless networks with user mobility," Queueing Systems, vol. 63, no. 1-4, pp. 131-164, 2009.

[16] L. Rong, S. E. Elayoubi, and O. B. Haddada, "Performance evaluation of cellular networks offering TV services," IEEE Trans. Veh. Technol., vol. 60, no. 2, pp. $644-655$, feb. 2011.

[17] M. K. Karray and M. Jovanovic, "A queueing theoretic approach to the dimensioning of wireless cellular networks serving variable bit-rate calls," IEEE Trans. Veh. Technol., vol. 62, no. 6, July 2013.

[18] J. Andrews, F. Baccelli, and R. Ganti, "A tractabl approach to coverage and rate in cellular networks," IEEE Trans. Commun., vol. 59, no. 11, pp. $3122-3134$, november 2011.

[19] H. S. Dhillon, R. K. Ganti, F. Baccelli, and J. G. Andrews, "Modeling and analysis of $k$-tier downlink heterogeneous cellular networks," IEEE J. Sel. Areas Commun., vol. 30, no. 3, pp. 550-560, 2012.

[20] B. Błaszczyszyn, M. Haenggi, P. Keeler, and S. Mukherjee, Stochastic Geometry Analysis of Cellular Networks. Cambridge University Press, 2018.

[21] R. Serfozo, Introduction to stochastic networks. Springer Science \& Business Media, 2012, vol. 44.

[22] F. Baccelli, B. Błaszczyszyn, and M. K. Karray, "Blocking rates in large CDMA networks via a spatial Erlang formula," in Proc. of IEEE INFOCOM, Miami, FL, USA, 2005.

[23] B. Błaszczyszyn and M. K. Karray, "Performance evaluation of scalable congestion control schemes for elastic traffic in cellular networks with power control," in Proc. of IEEE INFOCOM, May 2007.

[24] I. Siomina and D. Yuan, "Analysis of cell load coupling for 

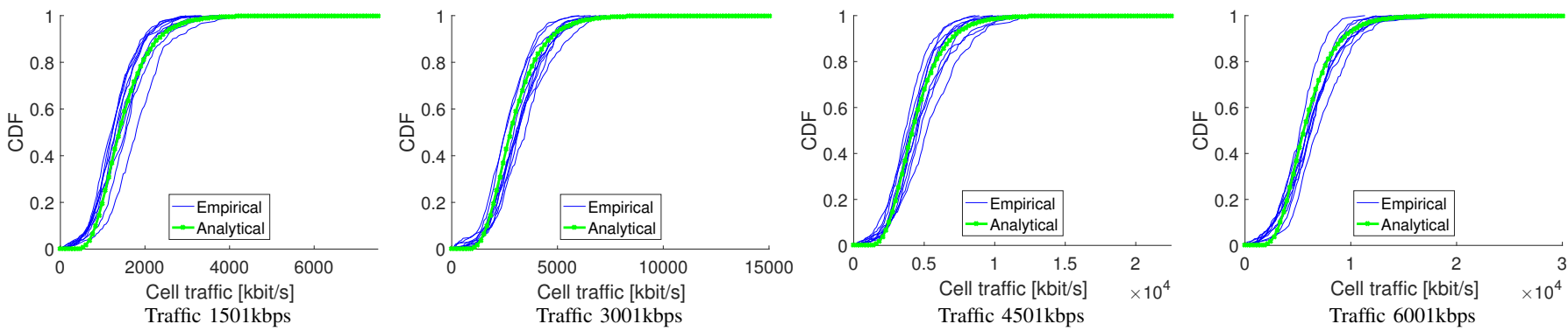

Fig. 5: Traffic demand, spatial distribution given some mean traffic demand: typical cell (Empirical) vs Gaussian approximate model (Analytic).
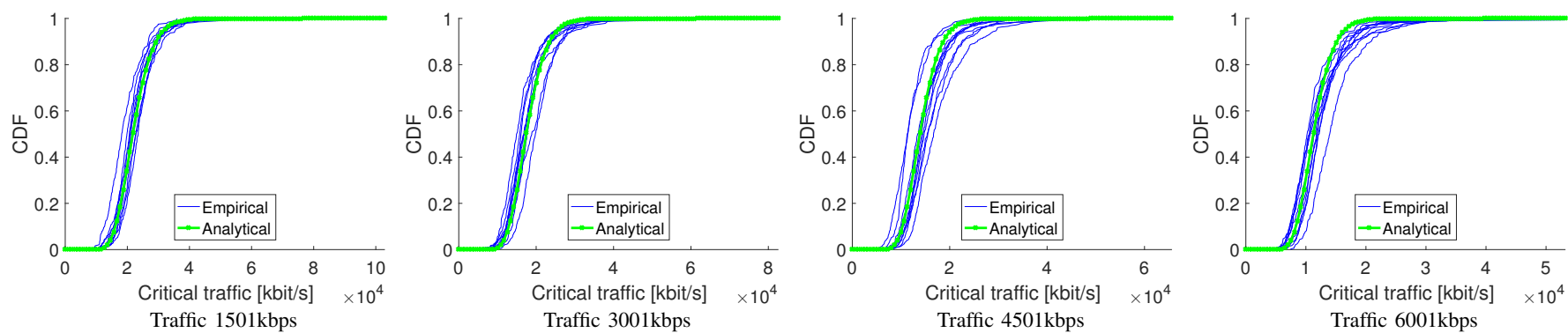

Fig. 6: Critical traffic demand, spatial distribution given some mean traffic demand: typical cell (Empirical) vs Gaussian approximate model (Analytic).
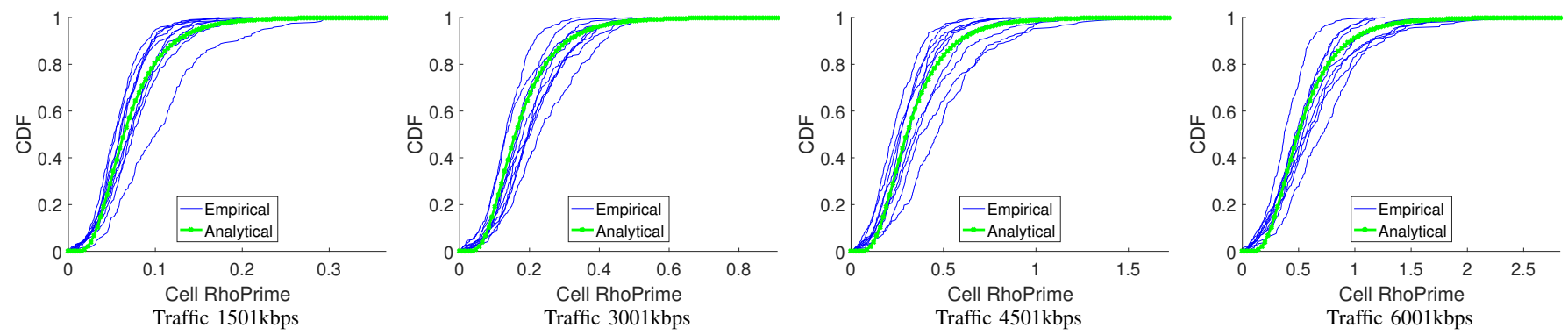

Fig. 7: Normalized traffic demand, spatial distribution given some mean traffic demand: typical cell (Empirical) vs Gaussian approximate model (Analytic).

LTE network planning and optimization," IEEE Trans. Wireless Commun., vol. 11, no. 6, pp. 2287-2297, 2012.

[25] Y. Zhou and W. Zhuang, "Performance analysis of cooperative communication in decentralized wireless networks with unsaturated traffic," IEEE Trans. Wireless Commun., vol. 15, no. 5, pp. 3518-3530, 2016.

[26] M. Foruhandeh, N. Tadayon, and S. Aïssa, "Uplink modeling of $k$-tier heterogeneous networks: A queuing theory approach," IEEE Commun. Lett., vol. 21, no. 1, pp. 164-167, 2017.

[27] Y. Zhong, T. Q. Quek, and X. Ge, "Heterogeneous cellular networks with spatio-temporal traffic: Delay analysis and scheduling," IEEE J. Sel. Areas Commun., vol. 35, no. 6, pp. 1373-1386, 2017.

[28] J. Tian, H. Zhang, D. Wu, and D. Yuan, "QoS-constrained medium access probability optimization in wireless interference-limited networks," IEEE Trans. Commun., 2017.

[29] P. Viswanath, D. N. C. Tse, and R. Laroia, "Opportunistic beamforming using dumb antennas," IEEE Trans. Inf. Theory, vol. 48, no. 6, pp. 1277-1294, 2002.

[30] A. Asadi and V. Mancuso, "A survey on opportunistic scheduling in wireless communications," IEEE Communications Surveys \& Tutorials, vol. 15, no. 4, pp. 1671-1688, 2013.

[31] F. Berggren and R. Jantti, "Asymptotically fair transmission scheduling over fading channels," IEEE Trans. Wireless Commun., vol. 3, no. 1, pp. 326-336, 2004.

[32] T. Ohto, K. Yamamoto, S.-L. Kim, T. Nishio, and M. Morikura,
"Stochastic geometry analysis of normalized SNR-based scheduling in downlink cellular networks," IEEE Wireless Commun. Lett., vol. 6, no. 4, pp. 438-441, 2017.

[33] K. Yamamoto, "Normalized SNR-based scheduling in Poisson networks," private communication, 2018.

[34] W. L. Tan, F. Lam, and W. C. Lau, "An empirical study on the capacity and performance of 3G networks," IEEE Transactions on Mobile Computing, vol. 7, no. 6, pp. 737-750, 2008.

[35] D. Willkomm, S. Machiraju, J. Bolot, and A. Wolisz, "Primary users in cellular networks: A large-scale measurement study," in New frontiers in dynamic spectrum access networks, 2008. DySPAN 2008. 3rd IEEE symposium on. IEEE, 2008, pp. 1-11.

[36] U. Paul, A. P. Subramanian, M. M. Buddhikot, and S. R. Das, "Understanding traffic dynamics in cellular data networks," in Proc. of IEEE INFOCOM. IEEE, 2011, pp. 882-890.

[37] M. Haenggi, "The meta distribution of the SIR in Poisson bipolar and cellular networks," IEEE Trans. Wireless Commun., vol. 15, no. 4, pp. 2577-2589, 2016.

[38] F. Baccelli and B. Błaszczyszyn, "A new phase transitions for local delays in MANETs," in Proc. of IEEE INFOCOM. IEEE, 2010, pp. 1-9.

[39] K. Majewski and M. Koonert, "Conservative cell load approximation for radio networks with shannon channels and its application to LTE network planning," in Telecommunications (AICT), 2010 Sixth Advanced International Conference on. IEEE, 2010, pp. 219-225.

[40] Z. Niu, Y. Wu, J. Gong, and Z. Yang, "Cell zooming for 

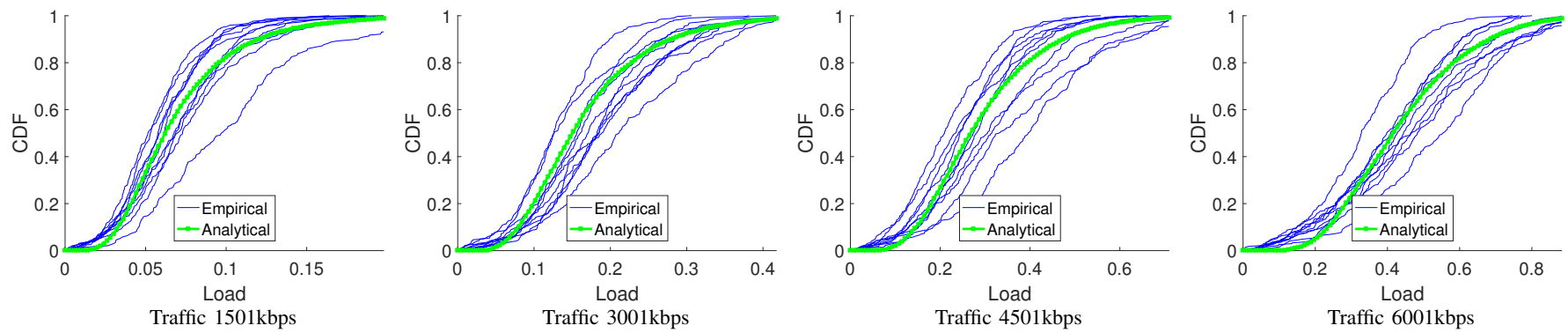

Fig. 8: Cell load, spatial distribution given some mean traffic demand: typical cell (Empirical) vs Gaussian approximate model (Analytic).
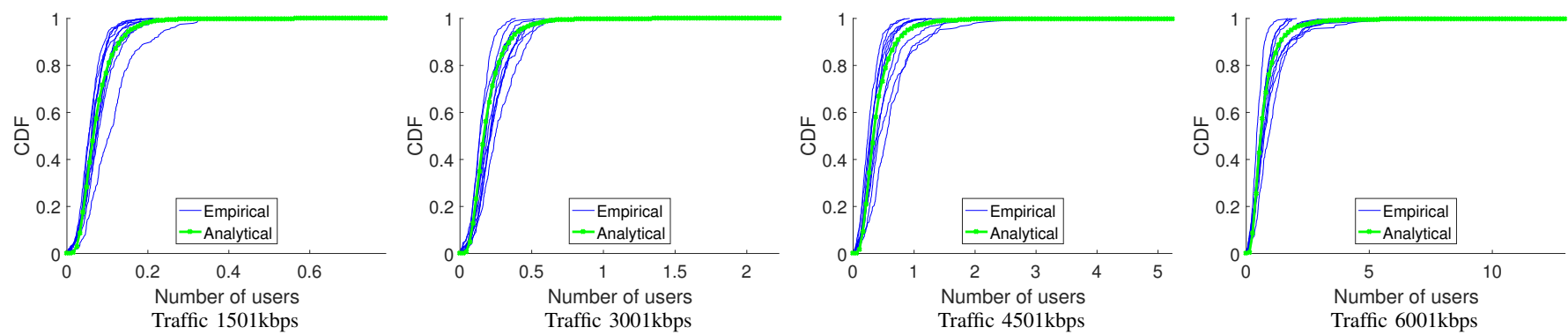

Fig. 9: Number of users, spatial distribution given some mean traffic demand: typical cell (Empirical) vs Gaussian approximate model (Analytic).
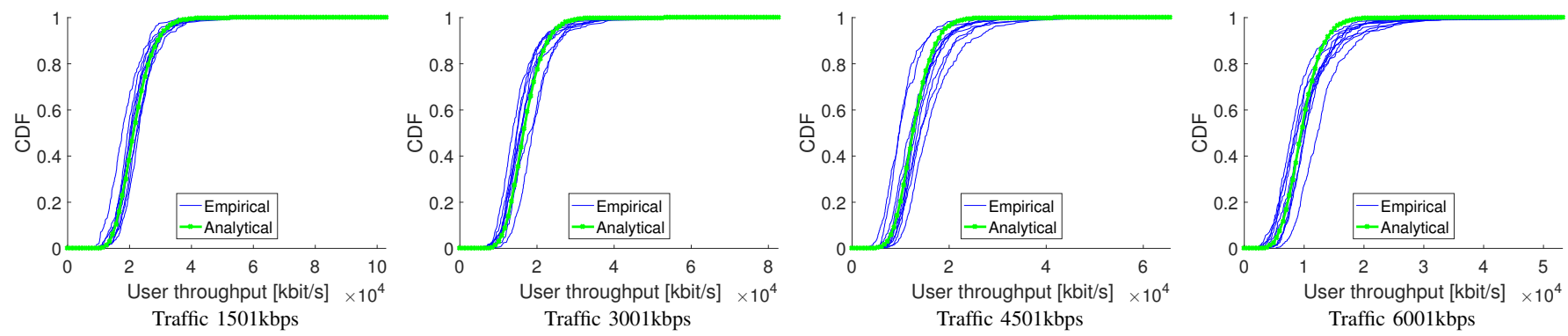

Fig. 10: User throughput, spatial distribution given some mean traffic demand: typical cell (Empirical) vs Gaussisn approximate model (Analytic).

cost-efficient green cellular networks," IEEE communications magazine, vol. 48, no. 11, 2010.

[41] H. Zhang, X. Qiu, L. Meng, and X. Zhang, "Design of distributed and autonomic load balancing for self-organization LTE," in Vehicular technology conference fall (VTC 2010-Fall), 2010 IEEE 72nd. IEEE, 2010, pp. 1-5.

[42] B. Błaszczyszyn, M. Jovanović, and M. K. Karray, "Performance laws of large heterogeneous cellular networks," in In proc. of WiOpt/SpaSWiN, 2015.

[43] B. Błaszczyszyn and M. K. Karray, "What frequency bandwidth to run cellular network in a given country? - a downlink dimensioning problem," in Proc. of WiOpt/SpaSWiN, 2015.

[44] M. Jovanovic, M. K. Karray, and B. Błaszczyszyn, "QoS and network performance estimation in heterogeneous cellular networks validated by real-field measurements," in Proc. of ACM PM2HW2N, Montreal, Canada, 2014.

[45] D. J. Daley and D. VereJones, An introduction to the theory of point processes. Volume I, 2nd ed. New York: Springer, 2003.

[46] I. E. Telatar, "Capacity of multiple antenna Gaussian channels," AT\&T Technical Memorandum, June 1995.

[47] J. W. Cohen, "The multiple phase service network with generalized processor sharing," Acta informatica, no. 3, pp. 245-284, 1979.

[48] F. P. Kelly, Reversibility and stochastic networks. Cambridge University Press, 2011.

[49] S. Borst, "User-level performance of channel-aware scheduling algorithms in wireless data networks," IEEE/ACM Transactions on Networking (TON), vol. 13, no. 3, pp. 636-647, 2005.

[50] F. Baccelli and B. Błaszczyszyn, Stochastic Geometry and Wireless Networks, Volume I - Theory, ser. Foundations and Trends in Networking. NoW Publishers, 2009, vol. 3, No 3-4.

[51] B. Błaszczyszyn, "Lecture Notes on Random Geometric Models - Random Graphs, Point Processes and Stochastic Geometry," 2017, http://hal.inria.fr/cel-01654766.

[52] H. P. Keeler, B. Błaszczyszyn, and M. K. Karray, "SINRbased k-coverage probability in cellular networks with arbitrary shadowing," in Proc. of IEEE ISIT, 2013.

[53] H. P. Keeler and B. Błaszczyszyn, "SINR in wireless networks and the two-parameter Poisson-Dirichlet process," IEEE Wireless Commun. Lett., vol. 3, no. 5, pp. 525-528, 2014.

[54] B. Błaszczyszyn and H. P. Keeler, "Studying the SINR process of the typical user in Poisson networks by using its factorial moment measures," IEEE Trans. Inf. Theory, 2015.

[55] I. Nakata and N. Miyoshi, "Spatial stochastic models for analysis of heterogeneous cellular networks with repulsively deployed base stations," Performance Evaluation, vol. 78, pp. 7-17, 2014.

[56] N. Miyoshi and T. Shirai, "Cellular networks with $\alpha$-Ginibre configurated base stations," in The Impact of Applications on Mathematics. Springer, 2014, pp. 211-226.

[57] N. Deng, W. Zhou, and M. Haenggi, "The Ginibre point process as a model for wireless networks with repulsion," IEEE Trans. Wireless Commun., vol. 14, no. 1, pp. 107-121, 2015. 